\title{
In ricordo di Antonio Zoppini
}

Il Prof. Antonio Zoppini è venuto a mancare nell'estate di quest'anno, dopo una lunga e dolorosa malattia. Il triste evento è stato vissuto in forma strettamente privata, ma è giusto ricordare il Prof. Zoppini a tutti coloro che Lo hanno conosciuto ed apprezzato all'interno del mondo accademico e ancor più ampiamente in ambito professionale, nell' arco della sua lunga carriera. E' altresì importante ricordarLo soprattutto a quei giovani reumatologi che non hanno avuto il privilegio di conoscerlo e frequentarlo direttamente.

Laureato in Medicina e Chirurgia presso l'Università degli Studi "La Sapienza" di Roma nel 1950, Specialista in Malattie dell'Apparato Respiratorio, Ematologia e Clinica Medica, Libero Docente in Reumatologia e Patologia Medica, la sua carriera è stata strettamente legata alla Scuola Reumatologica Romana. Diventato Assistente Ordinario e successivamente Aiuto presso la Cattedra di Reumatologia dell'Università degli Studi "La Sapienza" di Roma, dal 1972 è stato incaricato dell'insegnamento della Reumatologia presso l'Università degli Studi de L'Aquila dove, dal 1980 al 1983 è stato Professore Ordinario di Reumatologia. Dal 1983 al 1999 è stato Direttore dell'Istituto di Reumatologia e della Scuola di Specializzazione in Reumatologia dell'Università degli Studi "La Sapienza" di Roma.

La sua attività accademica è stata caratterizzata da un'ampia produzione scientifica su riviste mediche sia nazionali che internazionali e dalla stesura di diversi capitoli su trattati di in- teresse nazionale, facendosi particolarmente apprezzare nell' ambito dello studio delle artriti microcristalline e delle spondiloartriti. Valido clinico, aveva improntato tutta la sua attività, sia di docente che di medico, alla conoscenza e al trattamento delle patologie di interesse reumatologico, focalizzando la sua attenzione sui nuovi approcci farmacologici.

Uomo dai molti interessi, appassionato cacciatore, ma soprattutto amante della vita semplice e della natura, nel raro tempo libero amava recarsi nelle Marche, dove non solo si rilassava, ma recuperava forze e tranquillità, come amava ricordare ai suoi più stretti collaboratori. Carattere appassionato e schietto, dotato di molta ironia, persona umanamente sensibile e vera, attento alle necessità di chi lavorava con Lui, ma soprattutto presente e partecipe dei problemi dei pazienti ai quali si dedicava con professionalità e con grande umanità.

Negli ultimi anni era stato duramente provato dalla malattia e viveva ritirato nella tranquillità della sua casa circondato dagli affetti familiari. La sua morte ha lasciato in tutti coloro che lo hanno conosciuto ed apprezzato un senso di profonda tristezza e la consapevolezza di aver incontrato un Grande Uomo che ha affrontato con dignità esemplare le sfide che la vita gli ha presentato.

Guido Valesini, Antonio Spadaro Cattedra di Reumatologia, Univeristà di Roma "La Sapienza" 